\title{
Microbial arsenic oxidation and chemotaxis (Abstract)
}

\section{Gejiao Wang*, Kaixiang Shi}

State Key Laboratory of Agricultural Microbiology, College of Life Science and Technology, Huazhong Agricultural University, Wuhan, China

*Corresponding author: gejiao@mail.hzau.edu.cn

\begin{abstract}
We isolated a highly As(III) resistant [minimal inhibitory concentration (MIC) $=25$ $\mathrm{mM}$ ] and As(III)-oxidizing bacterium A. tumefaciens GW4 from As-enriched groundwater sediments. Unlike most of the heterotrophic As(III)-oxidizing bacteria using As(III) oxidation as a detoxification process, the As(III) oxidation of strain GW4 enhanced the bacterial growth, and the strain showed positive chemotaxis toward As(III) towards 0.5-2 mM As(III). Genomic analyses revealed a putative chemoreceptor-encoding gene, $m c p$, located in the arsenic gene island and having a predicted promoter binding site for the As(III) oxidation regulator AioR. Expression of $m c p$ and other chemotaxis related genes (cheA, cheY2 and fliG) was inducible by $\operatorname{As}(\mathrm{III})$, but not in the aioR mutant. Using capillary assays and intrinsic tryptophan fluorescence spectra analysis, Mcp was confirmed to be responsible for chemotaxis towards As(III) and to bind As(III) (but not As(V) nor phosphate) as part of the sensing mechanism. A bacterial one-hybrid system technique and electrophoretic mobility shift assays showed that AioR interacts with the $m c p$ regulatory region in vivo and in vitro, and the precise AioR binding site was confirmed using DNase I foot-printing. Taken together, these results indicate that this Mcp is responsible for the chemotactic response towards As(III) and is regulated by AioR. Additionally, disrupting the $m c p$ gene affected bacterial As(III) oxidation and growth, inferring that Mcp exerts functional connection between As(III) oxidation and As(III) chemotaxis.
\end{abstract}

Keywords: Chemotaxis, microbial arsenic oxidation 
